\title{
Towards the Development of a Frequency Agile MEMS Acoustic Sensor System
}

\author{
José Guerreiro, Andrew Reid, Joseph C. Jackson and James F.C. Windmill \\ Centre for Ultrasonic Engineering \\ University of Strathclyde \\ Glasgow, Scotland \\ jose.guerreiro@strath.ac.uk
}

\begin{abstract}
Designing acoustic sensors with adaptable frequency responses is of great interest in order to deal with diverse application requirements. A bio-inspired acoustic concept exploiting frequency agility using a MEMS microphone front-end is presented. Simulations and experimental results show adaptations of the microphone's acoustic frequency response according to applied DC voltage potentials. Finally, the microphone is demonstrated as part of an integrated adaptive frequency sensor feedback loop. Such acoustic sensor systems can be used in many applications requiring high frequency discrimination and agile tuning.
\end{abstract}

Keywords-bio-inspired acoustic transducer; frequency agility; MEMS microphone; feedback system; piezoelectric sensing; capacitive actuation; prototyping.

\section{INTRODUCTION}

Engineered acoustic and ultrasonic sensors are conventionally designed to be used with a rigid and fixed acoustic frequency response. The aim of this work is to create a sensor system which can be dynamically adaptable, for instance to cope with environmental demands or to operate within noisy scenarios, rather than rely on computationally expensive upper level signal processing stages. In biology, acoustic sensors have evolved to be adaptable and so developed feedback mechanisms that are able to process and compress acoustic information at the sensor periphery before sending it to higher level computational stages (i.e. the central nervous system). Clearly this gives advantages in terms of power efficiency, and the need to process a large amount of sensory information (e.g. sound) in a limited time frame [1]. Designing acoustic sensors such as MEMS microphones, which are inspired by nature is not new [2-4]. Moreover, a novel acoustic bio-inspired concept was recently presented in [5] where the "transducer becomes part of the signal processing chain". It can be achieved by exploring feedback mechanisms at the sensor level in order to alter its mechanical response and consequently its acoustic frequency response can be changed. Therefore, this paper aims to extend that study further by presenting a MEMS microphone designed to pursue the concept of a frequency agile transducer. It includes piezoelectric and capacitive sensing and actuation modalities that combine to allow this new device to be part of a feedback loop system in order to achieve signal processing capabilities at the microphone's structural level, while also using standard MEMS manufacturing techniques. Simulations and experimental results are presented in order to evaluate the new

This research is funded by the European Research Council under the European Union's Seventh Framework Programme (FP/2007-2013) / ERC Grant Agreement n. [615030]. device's acoustic frequency response under varying applied voltage potentials, and its integration within a feedback loop system.

\section{MICROPHONE DESIGN AND FABRICATION}

The new MEMS microphone comprises an $800 \mu \mathrm{m}$ radius diaphragm, $10 \mu \mathrm{m}$ thick membrane mounted on 6 serpentine spring arms. Each of the arms is coated with a $500 \mathrm{~nm}$ Aluminium Nitride layer for piezoelectric transduction, while the membrane itself is fringed by 176 (6 $\mu \mathrm{m}$ wide) capacitive combs, interdigitated with a stator bank of combs on the surrounding die at $6 \mu \mathrm{m}$ intervals, as shown in Fig. 1. The device is backside etched ( $400 \mu \mathrm{m}$ thick) at the moving parts to allow free release of movement.

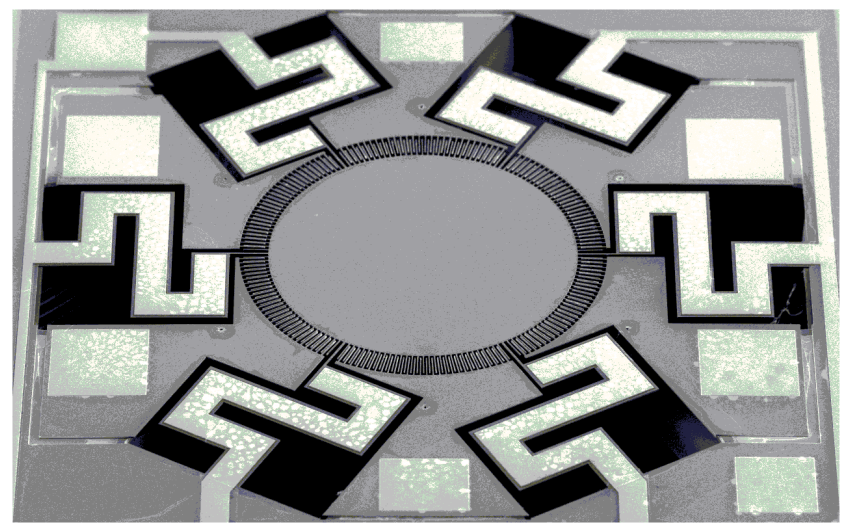

Fig. 1 - SEM of the device after fabrication (top view tilted $50^{\circ}-\mathrm{x} 30$ ).

The device was fabricated on single crystal Silicon-OnInsulator (SOI) using MEMSCAP's PiezoMUMPS service. In contrast to existing capacitive comb actuators and sensors the rotor combs here are intended to have a neutral position in plane with the stator combs, making the electromotive force from the biasing of the combs symmetrical about the $\mathrm{Z}$-axis. The relative thickness of the serpentine springs at multiple anchor points reduced the effect of through thickness stress gradients during fabrication to a negligible displacement around the centre of the membrane and the stator and rotor combs fully engaged.

\section{METHODS}

\section{A. Finite Element Modeling}

In order to predict the acousto-structural interactions in the MEMS microphone before its fabrication, finite element 
modelling using COMSOL Multiphysics was performed using the acoustics and structural mechanics modules. The rotational symmetry of the device allowed the model to be constructed as a $60^{\circ}$ segment of the membrane containing one spring arm and the associated stator and rotor combs surrounded by a cylindrical air domain, greatly reducing computation time. Simulating the effect of the bias voltage on the resonant frequency of the membrane required a moving mesh to be applied to the air domain. The minimum mesh element quality was determined to be greater than 0.1 with a minimum element size of $1 \mu \mathrm{m}$. A harmonic boundary load equivalent to $1 \mathrm{~Pa}$ was applied to the membrane surface and swept at $50 \mathrm{~Hz}$ intervals with varying bias voltages from 0 to $50 \mathrm{~V}$ applied to the rotor combs. The membrane damping was estimated as an applied loss factor of 0.015 , giving a $\mathrm{Q}$ of 30 .

The estimated resonance frequency in air for the membrane without bias was $3.15 \mathrm{kHz}$ increasing to $3.25 \mathrm{kHz}$ with a bias voltage of $50 \mathrm{~V}$. Displacement at resonance was estimated at $2.6 \mu \mathrm{m}$ for a $1 \mathrm{~Pa}$ load, however the net pressure from an acoustic wave is anticipated to be much lower due to the large diffraction around the membrane from a sound field at $3.0 \mathrm{kHz}$.
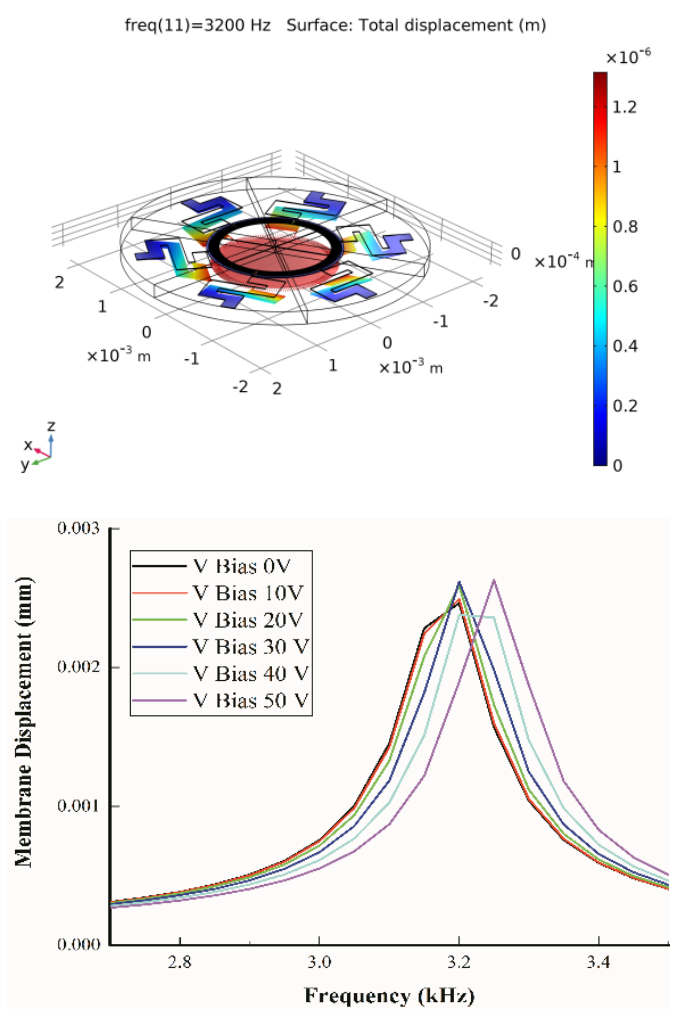

Fig. 2 - (Top) COMSOL simulation of membrane displacement at predicted resonance frequency of $3.20 \mathrm{kHz}$ in response to $1 \mathrm{~Pa}$ boundary load. (Bottom) Predicted resonant frequency shift with increasing bias voltage applied to rotor combs.

\section{B. Experimental Setup}

Initially, the device was positioned on a laser Doppler vibrometer (Polytec sensor head MSA-100-3D) stage to allow measurements of the motion on the microphone's diaphragm in response to sound. A sound transmitter (ESS HEIL Air-Motion Transformer) was placed perpendicular to the microphone, at a distance of $1 \mathrm{~m}$, and was excited by the internal signal generator of the laser vibrometer. Electrical signals from the microphone's piezoelectric port were amplified with a transimpedance amplifier (70dB of gain) and bandpass filtered $(2-12 \mathrm{kHz})$ through a custom-made analog circuit before being recorded with the acquisition laser vibrometer controller (Polytec frontend MSA-100-3D) with a sampling frequency of $50 \mathrm{kHz}$. The capacitive port of the microphone was directly driven by a DC voltage supply (from 0 to $50 \mathrm{~V}$ ). Finally, the MEMS microphone was positioned in a closed-loop setup in order to evaluate its adaptive capabilities in a real-time signal processing scenario.

\section{SIMULATED AND EXPERIMENTAL RESUlTS}

As previously described, the acousto-strutural interactions of the MEMS microphone were evaluated using finite element modelling in COMSOL as illustrated in Fig. 2. Simulations predicted an unbiased first resonance mode at approximately $3.15 \mathrm{kHz}$, which was measured using the laser Doppler vibrometer (LDV) at $3.34 \mathrm{kHz}$. The LDV measurements that were simultaneously recorded with the electrical signals from the piezoelectric port of the device for comparison are shown in Fig. 3A and B, respectively.

A
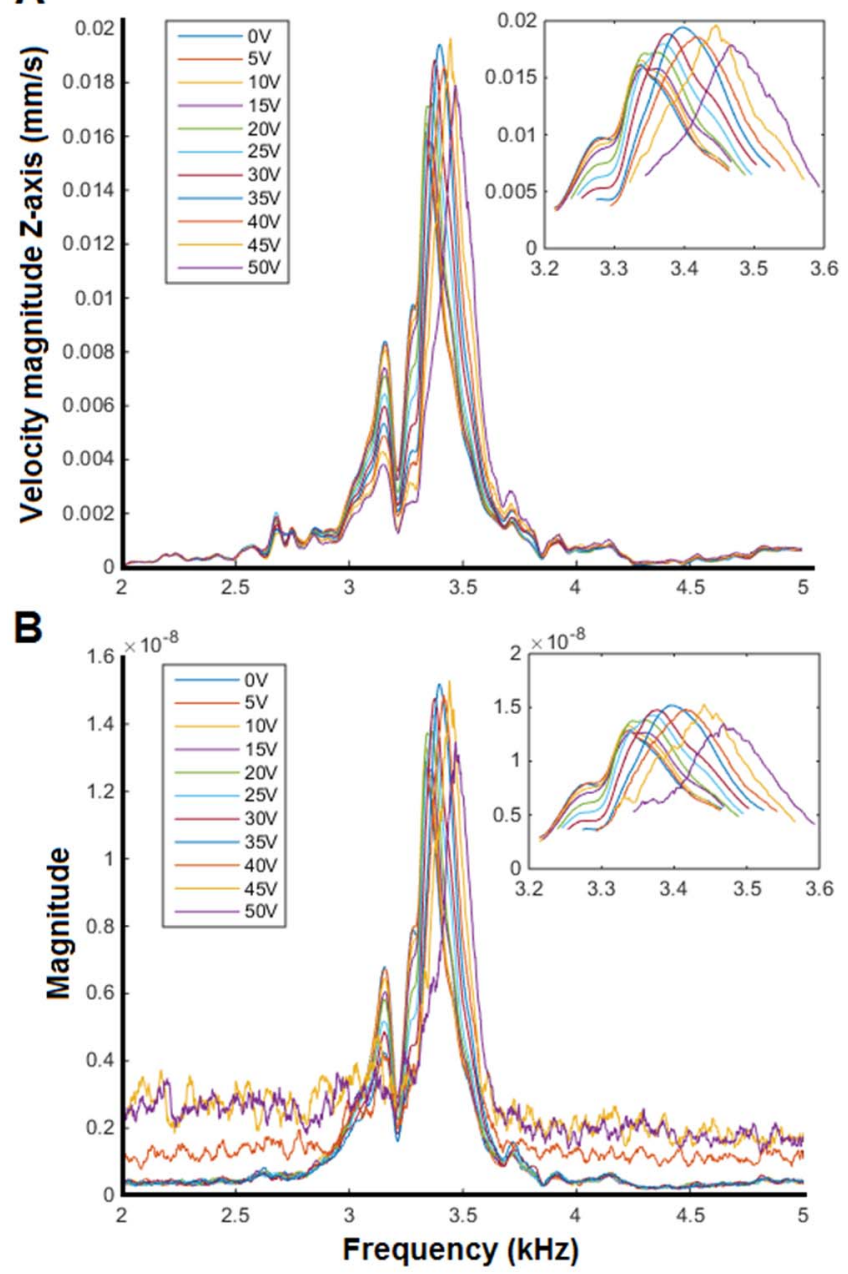

Fig. 3 - Frequency response of the microphone's diaphragm while applying different DC voltage potentials at the capacitive port of the device. (A) measured with LDV. (B) measured from the PZT sensing port. Inset graphs 
illustrate the zoom in between $3.2-3.6 \mathrm{kHz}$ that closely shows the change of the resonance frequency for different applied bias voltages.

As reported in the previous study [5], the simulated and measured acoustic frequency response of such a transducer can be altered by increasing its equivalent stiffness. Here, the stiffness increment effect over the microphone's diaphragm was tested by driving the capacitive port of the device with DC voltage potentials between 0 to $50 \mathrm{~V}$ with $5 \mathrm{~V}$ step increments. This resulted in an increment of the resonance frequency tested around its $1^{\text {st }}$ resonant mode, from $3.34 \mathrm{kHz}$ up to $3.47 \mathrm{kHz}$, respectively, as illustrated in Fig. 4.

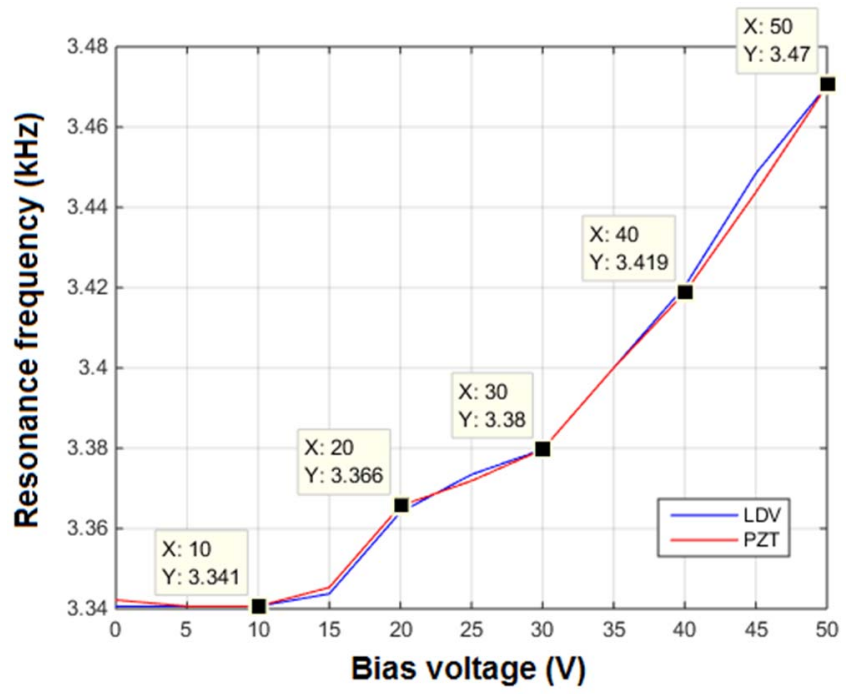

Fig. 4 - Resonance frequency shifting of the microphone's diaphragm when applying DC voltages from 0 - $50 \mathrm{~V}$ range. (blue) measured with LDV; (red) measured from the PZT sensing port.
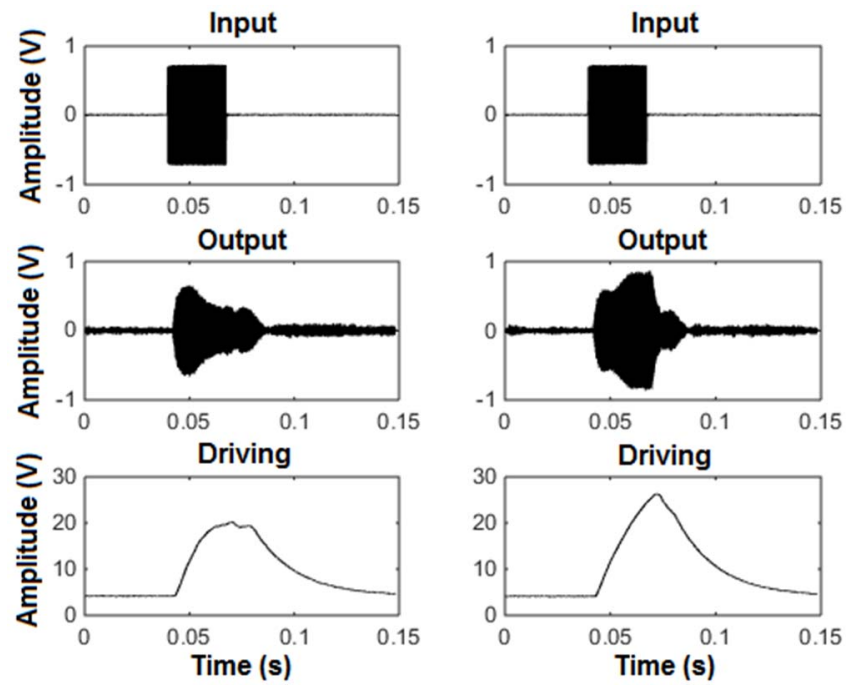

Fig. 5 - Dynamic adaptations of the MEMS microphone acoustic frequency response when placed in an closed-loop embedded system that provides results in real-time. (left) The microphone's resonance frequency is matched with the frequency of the input stimuli, however after $25 \mathrm{~ms}$ the device's frequency response becomes off-tuning while the DC bias is being continuously increased. (right) Resonance tunning response is seen matched with the input signal only after $25 \mathrm{~ms}$ of the DC bias start being uptaded.
Moreover, in order to evaluate the adaptive capabilities of this new device, it was placed in an embedded feedback loop system computing a threshold based algorithm to enable frequency agility of the front-end sensor (refer to [5] for complete description about the embedded system setup and algorithm used). Once a pre-defined threshold is reached, the embedded system automatically updates the bias voltage to the MEMS microphone (driving the rotor combs of the device) that consequently alters its acoustic frequency response. Fig. 5 shows two examples of frequency adaptation of the MEMS microphone given by the closed-loop embedded system. On the one hand, when acoustic input is presented to the system, the results show time $(25 \mathrm{~ms})$ and amplitude $(0.5 \mathrm{~V})$ dependencies given by the settings defined on the algorithm used. On the other hand, without any input stimuli the embedded system sets the bias voltage to return back to its initial value. Overall, the acoustic frequency response of this new sensor system (sensed by the piezoelectric port of the MEMS microphone) is changed dynamically as a function of the applied input sound level (acoustic stimuli) as similarly reported in the previous work [5].

\section{CONCLUSIONS}

This paper presents the design of a MEMS microphone with piezoelectric and capacitive sensing and actuation capabilities. Simulations and experimental results are presented and show that the device is able to alter its acoustic frequency response in correlation to a varied DC voltage potentials applied at the capacitive port. The resonance frequency ( $1^{\text {st }}$ resonant mode) can be shifted approximately within a $130 \mathrm{~Hz}$ bandwidth. Moreover, the real-time results show dynamic adaptations of the microphone's frequency response when placed in a closed-loop system. Future investigations based on this work would include further characterizations of the device and more evaluations of its behaviour when operated within the closed-loop system. The goal would be to provide frequency agility and adaptive tuning of a MEMS microphone sensor system in order to address realtime applications across diverse acoustic and ultrasonic fields.

\section{ACKNOWLEDGMENTS}

The authors would like to thank the staff and researchers of the Centre for Ultrasonic Engineering at the University of Strathclyde for all their support throughout this research work.

\section{REFERENCES}

[1] Sarpeshkar, R., "Ultra Low Power Bioelectonics: Fundamentals, Biomedical Applications, and Bio-inspired Systems". Cambridge University Press, 2010.

[2] Miles, R.N., Hoy, R.R., "The Development of a Biologically-Inspired Directional Microphone for Hearing Aids". Audiology Neurotology, 11(2): 86-94, 2006.

[3] Reid, A., Windmill, J.F.C., and Uttamchandani, D., "Bio-Inspired Sound Localization Sensor with High Directional Sensitivity”. Elsivier Procedia Engineering 120: 289-293, 2015.

[4] Zhang, Y., Bauer, R., Windmill, J.F.C., and Uttamchandani, D., "MultiBand Asymmetric Piezoelectric MEMS Microphone Inspired by the Ormia Ochracea". IEEE $29^{\text {th }}$ International Conference on MEMS, 1114$1117,2016$.

[5] José Guerreiro, Joseph C. Jackson and James F.C. Windmill. "Simple Ears Inspire Frequency Agility in an Engineered Acoustic Sensor System". IEEE Sensors Journal, 2017. 